\title{
Delayed infection, family size and malignant lymphomas
}

P Vineis, L Miligi, P Crosignani, A Fontana, G Masala, O Nanni, V Ramazzotti, S Rodella, E Stagnaro, R Tumino, C Viganò, C Vindigni, A Seniori Costantini

\begin{abstract}
Background-The annual incidence of non-Hodgkin's lymphomas (NHL) is increasing by $3 \%-4 \%$ in different parts of the developed world. Excesses of NHL have been observed in populations exposed to immunosuppressants and to HIV, but these causes do not explain the increasing trends. It is suggested that delayed infection could explain NHL trends, through an impairment of the Th1/Th2 lymphocyte patterns.
\end{abstract}

Methods-In a population-based study on 1388 patients with NHL, 354 with Hodgkin's disease (HD) and 1718 healthy controls, the age of first occurrence of bacterial and viral diseases was investigated. Clinical records were perused in one centre to check the anamnestic data.

Findings-The age of occurrence of bacterial and viral diseases was significantly higher among NHL patients than in the controls. The association between later age at first bacterial or viral disease was limited to small families $(O R=1.95 ; 95 \%$ confidence intervals $1.26,3.00$, for age $4-8$ at first infection; $O R=1.91 ; 1.19,3.06$, for age 9+, compared with less than 4 ). The association was more obvious for bacterial diseases (possibly for the lower degree of misclassification). High grade lymphomas showed the strongest association. The later age of occurrence of bacterial or viral diseases in NHL patients is consistent with a higher incidence of lymphomas observed in higher social groups. No clear association was found between HD and age at first bacterial or viral diseases.

Interpretation-It is proposed that delayed infection could explain the increasing NHL trends, through an impairment of the Th1/Th2 lymphocyte patterns. The model of delayed infection has been proposed also to explain increasing prevalence rates of asthma.

Institute, Genova, Italy E Stagnaro

Cancer Registry, Ragusa, Italy R Tumino

Department of Pathology, University of Siena, Italy C Vindigni

Correspondence to: Dr Vineis

(paolo.vineis@unito.it)

Accepted for publication 14 June 2000

The aetiology of lymphomas is still largely unknown. The known risk factors explain only a small proportion of the cases that occur in the world. ${ }^{1-9}$ Time trends in recent decades show stable rates for Hodgkin's disease (HD), but a considerable increase of the incidence rates for non-Hodgkin's lymphomas (NHL) in most Western countries. The increase in NHL cannot be explained by the AIDS epidemic, being larger than justified by the strength of association with the HIV infection. The increase is real and not artefactual-that is, attributable to improved diagnostic ability. ${ }^{10}$ Within the SEER programme in the USA, the strongest increments in time trends for NHL in 1978-1988 occurred for high grade NHL (15\% per year, versus 3\% among low grade and $2 \%$ among intermediate grade NHL). ${ }^{1}$ The fact that highly malignant grades show the most evident increase confirms that the trend is not an artefact attributable to increased diagnostic sensitivity.

Well established risk factors for NHL are immunosuppression in transplanted patients (with a 100-fold increased risk) and in HIV infected patients. ${ }^{9}$ The causes of HD are more uncertain. ${ }^{11}$ It is reasonable to believe that both malignancies are related to impairment of the immune system in response to external stimuli. Human immune system responses are heterogeneous and may involve antagonism between T helper (Th) lymphocyte subsets (Th1 and Th2) and their cytokines. A pathogenetic role of interleukin (IL) 10 in NHL has been proposed. IL10 has a strong immunosuppressive action via inhibition of Th1 type cytokines. ${ }^{12}$ A polarised Th1 response has been invoked in the pathogenesis of several infectious diseases, and also of peptic ulcer after infection from Helicobacter pylori. ${ }^{13}$ A positive reaction to tuberculin was associated with a cytokine profile biased towards Th1, and predicted a lower incidence of asthma. ${ }^{14}$ Allergic respiratory diseases such as bronchial asthma are believed to result from the repeated expression in airway tissues of Th2 polarised $\mathrm{T}$ cell immunity to inhaled allergens. The Th1/ Th2 switching process is influenced by a number of host and environmental factors that are poorly understood. Prominent among these are factors that affect the kinetics of maturation of immune competence during the early postnatal period. It is hypothesised that the principal impetus for postnatal development of a normal Th1/Th2 balance is provided via contact with Th1 stimulatory micro-organisms at the body's major mucosal surfaces. ${ }^{15} 16$

Several diseases have been characterised as showing a Th1 dominant or Th2 dominant lymphocyte pattern. In the first category rheumatoid arthitis, multiple sclerosis, sarcoidosis and diabetes have been included, ${ }^{17}{ }^{18}$ while the second comprises Crohn's disease, ulcerative colitis, allergies (including hay fever), asthma, atopies (including eczema), basal cell carcinoma, Sézary's syndrome, EBV associated NHL, HD, and large cell anaplastic lymphoma.

Several similarities between lymphomas (particularly HD) and some of the diseases that 
have been mentioned are notable. In developing countries there is a peak for HD in young age, while in the developed countries there is a peak in young adults. In analytical studies, younger children (less than 10) with HD tend to belong to lower social groups ( $\mathrm{OR}=3.6$ in Gutensohn and Shapiro, 1982), ${ }^{19}$ while older children (1014) did not show social class differences from their controls. This may reflect a shift in age of exposure to common infections. Young adults with HD tend to belong to higher social groups (for example Cohen et al, 1964; Gutensohn and Cole, 1977: $\mathrm{OR}=2.6),{ }^{21}$ and persons belonging to the largest families have a risk that is half of persons from the smallest families. In one study cases tended to have had fewer playmates as a child, and the parents were more educated than controls. ${ }^{21}$ Middle age cases appeared to be people whose childhood provided some protection from early infection. ${ }^{11}$ There is little to support the thesis that HD is a transmissible disease, transmitted by person to person contact. This, however, is not inconsistent with an infectious aetiology. For example, subacute sclerosing panencephalitis is infectious (it is an age dependent consequence of measles infection), but is not contagious. The role played by infection in HD is also suggested by the association with tonsillectomy, which seems to be related to family size. ${ }^{22}$ Tonsillectomy seems to be also a risk factor for paralytic poliomyelitis ${ }^{23}$ and multiple sclerosis. ${ }^{24}$

Much less is known about infectious diseases during childhood and NHL. However, asthma is increasing in developed countries at a rate that is similar to that for NHL. ${ }^{25}$ One explanation that has been proposed is that such an increase is attributable to delayed exposure to viruses and bacteria, in comparison with developing countries and with past decades in developed countries. Such delayed exposure would alter the maturation of the immune system, based on a shift from a predominantly Th2 response to a Th1 response pattern during childhood. Parallels between asthma and NHL are worth exploring. Recently, a study in Germany has suggested that asthma can be related to later exposure to infectious agents attributable to delayed entry to day nursery. ${ }^{26}$ Day care children have more infections-in particular diarrhoea, hepatitis A, otitis media, respiratory tract infections and haemophilus influenza. ${ }^{27}{ }^{28}$ The findings in German children support the hypothesis that early infection may protect from allergies through stimulation of Th1 lymphocytes, which may inhibit the expansion of antigen specific Th2 lymphocytes.

We have tested the hypothesis of a relation between delayed infection and the risk of lymphomas in Western countries.

\section{Methods}

We have conducted a population-based study in Italy. In each of 11 areas, all the subjects suspected of being affected by lymphoma were identified through periodical surveys in the hospital departments where such cases are diagnosed. Only newly diagnosed cases occurring in the study period were included (both sexes, aged 20-74, residents of the areas under study). For case classification-based on immunocytochemical techniques-we used the Working Formulation for NHL. ${ }^{29}$ The control group was formed by a random sample of the population resident in each of the areas, aged 20-74. The sample was stratified according to five year age groups and sex, and its size was equal to the number of cases in the largest diagnostic group (NHL+CLL). Procedures for control sampling were of two types. In the provinces of Forli and Ragusa and in the city of Firenze the computerised and regularly updated demographic files were used. These files are maintained under national law in all cities and towns throughout Italy. They are central population registries that serve such multiple purposes as voter registration, car insurance, school eligibility, and pensions. They are well validated as epidemiological data sources, are continually updated, and highly accurate. In the other areas (where the demographic files were not accessible to the investigators) sampling took place through the files of the National Health Service, which are updated every six months.

Information about the known or suspected risk factors for the diseases under investigation was collected through person to person interviews. Techniques for the motivation of subjects to participate, such as contacts with the general practitioners, were applied. The personnel in charge of the interviews was trained specifically for this study; the interview lasted about one hour. Interviews were generally conducted at home and were blind as to the casecontrol status and to the hypotheses under investigation. We have investigated the entire medical history for the following diseases: measles, rubeola, chickenpox, pertussis, herpes zoster, herpes labialis, herpes genitalis, mumps, infectious hepatitis, infectious mononucleosis, tuberculosis, malaria, other infectious diseases (specified by the interviewee), hay fever, allergic asthma, other (specified) allergies, peptic ulcer, ulcerative colitis, glutine intolerance, Crohn's disease, other (specified) digestive diseases, diabetes and other (specified) metabolic diseases, rheumatoid arthritis, lupus erythematosus, periarteritis nodosa, sclerodermia, other (specified) osteomuscular diseases, urticaria, eczema, psoriasis, other skin allergies, and other (specified) relevant diseases. Only diseases that had been formally diagnosed by a physician were considered. For each disease the date of occurrence was investigated, except for those under the label "other, specified". The overall refusal rate was $10 \%$ among cases and $19 \%$ among controls.

Further details on methods, and complete data on the associations between disease history and the risk of haematopoietic malignancies are given in a previous paper. ${ }^{30}$

QUALITY CHECKS AND STATISTICAL ANALYSES In addition to analysing interviews, in the Torino centre we have perused 52 clinical records corresponding to NHL or HD cases who had reported peptic ulcer $(n=30)$ or hepatitis $(n=22)$ (as controls were sampled from the 
population, a clinical record was not easily accessible). Twenty one clinical records, out of 30 , mentioned peptic ulcer, and 13 of 22 mentioned hepatitis. Age at first infection was collected from clinical records.

The average age of first viral or bacterial disease (among those considered), and the average age of the last episode have been computed, after exclusion of the last five years before the diagnosis of lymphoma or the interview of control subjects. Viral diseases were infectious mononucleosis, measles, rubeola, chickenpox, herpes (zoster, labialis, genitalis) and hepatitis; bacterial diseases were pertussis, tuberculosis, peptic ulcer and other diseases specified by the interviewee. Age at onset has been checked in the 52 clinical records perused in Torino.

Regression coefficients have been estimated in unconditional logistic regression models, adjusted for gender and the age at lymphoma diagnosis or at interview of controls. Odds ratios and Wald 95\% confidence limits (CI) have been computed from logistic parameter estimates. $^{31}$

Analyses have been repeated after discarding the interviews lasting less than 40 minutes, incomplete interviews and interviews whose quality was judged less than satisfactory by the personnel (best evidence criterion).

As a separate exercise, we have also considered the world distribution of asthma and lymphomas. Prevalence rates of asthma among male children (life long and in the past 12 months) has been collected for 64 countries in the world from a publication of the ISAAC Working Group. ${ }^{32}$ For 35 of these 64 countries

Table 1 Sibship size (not including the index subject) among population controls $(n=1718)$ and cases affected by non-Hodgkin's lymphomas $(n=1388)$ or Hodgkin's disease $(n=354)$. Data are age adjusted odds ratios and $95 \%$ confidence intervals in parentheses

\begin{tabular}{lll}
\hline Sibship size & Men & Women \\
\hline Non-Hodgkin's lymphomas & \\
$0^{\star}$ & 1.0 & 1.0 \\
1 & $0.89(0.60,1.31)$ & $1.03(0.67,1.59)$ \\
$2-3$ & $0.88(0.59,1.32)$ & $1.02(0.67,1.58)$ \\
$4+$ & $0.91(0.63,1.31)$ & $0.94(0.63,1.39)$ \\
Hodgkin's lymphomas & \\
$0^{\star}$ & 1.0 & 1.0 \\
1 & $0.88(0.45,1.71)$ & $0.97(0.51,1.83)$ \\
$2-3$ & $1.45(0.72,2.92)$ & $1.02(0.53,1.97)$ \\
$4+$ & $0.89(0.46,1.73)$ & $0.60(0.31,1.16)$ \\
\hline
\end{tabular}

${ }^{\star}$ Reference category.

Table 2 Number of subjects by age at first occurrence of infectious diseases * among population controls $(n=1718)$ and among cases affected by non-Hodgkin's lymphomas (NHL) (n=1388) or Hodgkin's disease (HD) ( $n=354)$, according to sibship size (not including the index subject). All infections in the five years preceding the diagnosis of lymphoma or the interview of controls have been excluded. Age unknown at first infection excluded

\begin{tabular}{|c|c|c|c|c|c|c|c|c|c|}
\hline \multirow[b]{3}{*}{ Sibship size } & \multicolumn{9}{|c|}{ Age at first infection } \\
\hline & \multicolumn{3}{|c|}{ Controls } & \multicolumn{3}{|c|}{$N H L$} & \multicolumn{3}{|l|}{$H D$} \\
\hline & $<4$ & $4-8$ & $9+$ & $<4$ & $4-8$ & $9+$ & $<4$ & $4-8$ & $9+$ \\
\hline 0 & 28 & 57 & 39 & 13 & 55 & 38 & 13 & 15 & 8 \\
\hline 1 & 66 & 166 & 106 & 24 & 126 & 94 & 19 & 45 & 26 \\
\hline $2-3$ & 52 & 253 & 170 & 40 & 159 & 122 & 21 & 55 & 28 \\
\hline $4+$ & 42 & 220 & 189 & 35 & 141 & 181 & 9 & 28 & 24 \\
\hline Totals & 188 & 696 & 504 & 112 & 481 & 435 & 62 & 143 & 86 \\
\hline Age unknown & \multicolumn{3}{|c|}{$240(14 \%)$} & \multicolumn{3}{|c|}{$259(19 \%)$} & \multicolumn{3}{|c|}{$41(12 \%)$} \\
\hline Information not available & \multicolumn{3}{|c|}{$90(5 \%)$} & \multicolumn{3}{|c|}{$101(7 \%)$} & \multicolumn{3}{|c|}{$22(6 \%)$} \\
\hline
\end{tabular}

${ }^{\star}$ List of diseases in the text.
Table 3 Age at first occurrence of infectious diseases * among population controls $(n=1718)$ and cases affected by non-Hodgkin's lymphomas $(n=1388)$, according to sibship size (excluding the index subject) and by education (years of school). All infections in the five years preceding the diagnosis of lymphoma or the interview of controls have been excluded. Age unknown at first infection excluded. Gender and age adjusted odds ratios, and 95\% confidence intervals in parentheses

\begin{tabular}{llll}
\hline \multirow{4}{*}{ Age at first occurrence } \\
\cline { 2 - 4 } Sibship size & $<4$ & $4-8$ & $9+$ \\
\hline 0 & 1.0 & $1.77(0.78,3.98)$ & $1.50(0.62,3.62)$ \\
1 & 1.0 & $1.98(1.17,3.32)$ & $2.08(1.16,3.72)$ \\
$0-1$ & 1.0 & $1.95(1.26,3.00)$ & $1.91(1.19,3.06)$ \\
$2-3$ & 1.0 & $0.81(0.51,1.29)$ & $1.01(0.63,1.63)$ \\
$4+$ & 1.0 & $0.70(0.42,1.15)$ & $1.04(0.61,1.75)$ \\
Education & years of school) & \\
$<9$ & 1.0 & $1.01(0.72,1.43)$ & $1.18(0.83,1.68)$ \\
$9-14$ & 1.0 & $1.16(0.70,1.93)$ & $1.39(0.81,2.38)$ \\
$15+$ & 1.0 & $1.43(0.69,2.96)$ & $2.50(1.18,5.32)$ \\
\hline
\end{tabular}

$\star$ The list of diseases is in the text.

Table 4 Age at first occurrence of bacterial diseases * among population controls $(n=1718)$ and cases affected by non-Hodgkin's lymphomas $(n=1388)$, according to sibship size (excluding the index subject). All infections in the five years preceding the diagnosis of lymphoma or the interview of controls have been excluded. Age unknown at first infection excluded. Gender and age adjusted odds ratios, and $95 \%$ confidence intervals in parentheses

\begin{tabular}{llll}
\hline \multirow{4}{*}{ Sibship size } & $<4$ & $4-8$ & \\
\cline { 2 - 4 } & $<+$ & $9+$ \\
\hline 0 & 1.0 & $1.20(0.29,4.92)$ & $2.13(0.50,8.95)$ \\
1 & 1.0 & $2.86(1.35,6.02)$ & $1.78(0.87,3.67)$ \\
$0-1$ & 1.0 & $2.50(1.33,4.79)$ & $2.00(1.08,3.70)$ \\
$2-3$ & 1.0 & $1.47(0.72,3.01)$ & $1.22(0.61,2.41)$ \\
$4+$ & 1.0 & $0.61(0.28,1.32)$ & $1.02(0.44,2.33)$
\end{tabular}

$\star$ The list of diseases is in the text.

age adjusted annual incidence rates (per 100000 residents) for NHL and HD in men have been identified from IARC's Cancer Incidence in Five Continents. ${ }^{4}$ We have computed correlation coefficients for the association between rates of lymphoma and rates of asthma.

\section{Results}

Table 1 shows the relations between the sibship size and the risk of NHL or HD. Only a weak association between large sibships and the risk of NHL is appreciable, but none of the odds ratios is statistically significant.

Table 2 gives the distribution of cases and controls by sibship size and age at first viral or bacterial disease. Cases and controls who did not recall the date of first viral or bacterial disease were excluded. Table 3 shows the gender and age adjusted odds ratios and $95 \%$ CI for NHL, according to the sibship size or educational level and age at first infectious disease. Small families show increased and statistically significant odds ratios associated with delayed infectious diseases. Also, odds ratios for delayed infection are higher for higher social groups (based on the educational level), with an odds ratio of 2.5 among those with more than 14 years of school.

Table 4 gives similar information, but only for bacterial diseases. The association with delayed infection is again particularly evident in small families (0-1 sibs). 
Table 5 Age at first occurrence of infectious diseases * among population controls $(n=1718)$ and cases affected by Hodgkin's lymphomas $(n=354)$, according to sibship size (excluding the index subject) and by education (years of school). All infections in the five years preceding the diagnosis of lymphoma or the interview of controls have been excluded. Age unknown at first infection excluded. Gender and age adjusted odds ratios, and 95\% confidence intervals in parentheses

\begin{tabular}{llll}
\hline \multirow{4}{*}{ Age at first occurrence } \\
\cline { 2 - 4 } Sibship size & $<4$ & $4-8$ & $9+$ \\
\hline $0-1$ & 1.0 & $1.0(0.57,1.67)$ & $1.17(0.62,2.23)$ \\
$2-3$ & 1.0 & $0.44(0.22,0.87)$ & $0.60(0.29,1.24)$ \\
$4+$ & 1.0 & $0.64(0.26,1.54)$ & $0.66(0.26,1.68)$ \\
Education (years of school) & \\
$<9$ & 1.0 & $0.57(0.31,1.04)$ & $0.73(0.39,1.37)$ \\
$9-14$ & 1.0 & $0.84(0.48,1.48)$ & $0.89(0.45,1.75)$ \\
$15+$ & 1.0 & $0.84(0.37,1.95)$ & $0.96(0.30,3.10)$ \\
\hline
\end{tabular}

*The list of diseases is in the text.

An association between the NHL grade (Working Formulation) and delayed infectious diseases was also observed: when considering only small families (sibship size $0-1$ ), the odds ratios for high grade versus low-intermediate grades were $2.09(0.68,6.40$; age $4-8)$ and 2.00 $(0.65,5.99$; age $9+$, versus age less than 4$)$.

According to a logistic regression model, the age adjusted parameter estimate for the age at first viral or bacterial disease as a continuous variable was 0.012 for NHL (standard error $0.003 ; p$ value $=0.0018$ ). This suggests that each one year increment in the delay of infections would result in a $1.2 \%$ increase in the risk of NHL. Logistic regression models including educational level (three groups), sibship size and gender as covariates gave virtually identical estimates.

A detailed analysis of HD was hampered by smaller numbers. In addition, estimates for HD were strongly confounded by age at HD diagnosis. The general pattern suggests only a weak association with late infectious diseases, if any (table 5).

Concerning quality checks, the average age at onset for the NHL cases with hepatitis whose clinical records were traced, was 25.4 (SE 4.9), versus 25.9 (1.5) among all cases, and 22.8 (1.4) among controls. For peptic ulcer the age of onset was 41 (SE 7.0) for cases whose clinical record was traced, 37.4 (2.4) for all cases, and 36.1 (1.0) for controls. The criterion of "best evidence" supports our observation. After discarding one third of interviews of lower quality, the odds ratios for NHL and delayed infectious diseases (sibship size $0-1$ ) still were $1.63(0.91,2.92$; age $4-8)$ and 1.80 $(1.00,3.30$; age $9+)$. Two categories of bacterial diseases had low rates of unkown dates of onset, peptic ulcer ( $H$ pylori infection) and "other, specified diseases" $(2.1 \%$ of unknown dates among controls, 3.9\% among NHL). Among these, the odds ratios for NHL in men, sibship size $0-1$, were $3.67(0.13,106$; age 4-8) and 2.29 (0.28, 18.38; age 9+).

Finally, we have computed correlation coefficients between lymphoma incidence rates in men and prevalence rates of asthma among male children in 35 countries. The correlation coefficient was $0.40(p=0.017)$ for NHL and $0.06(p=0.72)$ for Hodgkin's lymphoma when
KEY POINTS

- The annual incidence of non-Hodgkin's lymphomas (NHL) is increasing by $3-4 \%$ in different parts of the developed world but the reasons are unknown.

- In a population-based study on 1388 patients with NHL, 354 with Hodgkin's disease (HD) and 1718 healthy controls, the age of first occurrence of bacterial and viral diseases was significantly higher among NHL patients than in the controls.

- The association between later age at first bacterial or viral disease was limited to small families $(\mathrm{OR}=1.95$, $95 \%$ CI $1.26,3.00$, for age $4-8$ at first infection; $\mathrm{OR}=1.91,95 \%$ CI 1.19 , 3.06, for age $9+$, compared with less than 4). The association was more obvious for bacterial diseases.

- We propose that delayed infection could explain the increasing NHL trends, through an impairment of the Th1/Th2 lymphocyte patterns. The model of delayed infection has been proposed also to explain increasing prevalence rates of asthma.

considering the 12 month prevalence rate of asthma, and $0.39(0.02)$ and -0.07 (0.67), respectively, when considering "ever having had asthma".

\section{Discussion}

We have observed, in a large population-based study, an association between the risk of NHL (but not HD) and a later age at onset of common infectious diseases, limited to small size families. Our finding is consistent with current hypotheses on the development of the immunological system, particulary the Th1/ Th2 switching process. The main pitfall of the study is that it relies upon interviews; however, checks of clinical records were made, which confirmed the anamnestic data. The fact that the association concerned mainly bacterial diseases may reflect a lower degree of random misclassification for these infections, which are less common and usually more severe then viral diseases, are treated with antibiotics, and are therefore easier to recall.

It is unlikely that the finding is attributable to recall bias. Evenly distributed inaccuracies would tend to bias the association towards the null, while a more accurate recall by the controls (that is, recall of earlier dates of onset of infectious diseases) is highly unlikely. The observed role of small family size and the lack of association between HD and age at first infection also makes recall bias an unlikely explanation. Restriction of the analyses to the interviews of higher quality did not change the results. Also the fact that the association was particularly strong among high grade lymphomas reinforces our hypothesis, as high grade lymphomas are those that increased more steeply in recent years. In addition, the 
association between age at first infectious disease and NHL showed a gradient with social class (educational level), with an odds ratio of 2.5 (95\% CI 1.18, 5.32) among those with more than 14 years of school. This finding, again, makes recall bias an unlikely explanation.

It is rather difficult to imagine how the present hypothesis could be confirmed with a different study design, unless large cohort studies in children are carried out with detailed information on infectious diseases, not affected by recall bias. Even so, the incidence of lymphomas would be extremely low and the study would require decades to be completed. Perhaps serum banks in which the prevalence of the antibody response to different infectious agents has been measured at different ages could be used as proxies.

Our finding is consistent with previous theories concerning the role of immunological changes (particularly in the Th1/Th2 balance), which take place early in life and can be involved in diseases like asthma. A study in Germany has suggested that asthma can be related to later exposure to infectious agents attributable to delayed entry to day nursery. ${ }^{26}$ This relation was present only in small families. A large sibship size was found to protect from asthma also in a study in New Zealand. ${ }^{33}$ It might not be incidental that both asthma and NHL are rapidly increasing in Western countries. We have also observed a highly statistically significant correlation between asthma in children and NHL (but not HD) incidence rates at a world level.

Interestingly, a later onset of infection has been invoked also to explain the aetiology of multiple sclerosis, a disease that shares many epidemiological features with lymphomas. Although most persons are infected asymptomatically with EBV in childhood, infection in adolescence or early adulthood can cause clinical infectious mononucleosis, a history of which greatly increases (sevenfold) the risk of multiple sclerosis. ${ }^{34}$

\section{Contributors}

Paolo Vineis and Adele Seniori coordinated and designed the study. Lucia Miligi was involved in the study design and statistical analyses. All the investigators listed in the Working Group on cal analyses. All the investigators listed in the Working Group on the Epidemiology of Hematolymphopoietic Malignancies in ing interviews.

Funding: this work has been funded by the American National Cancer Institute (grant no NCI CA51086), by the European Community (Europe Against Cancer Programme) and by the Community (Europe Against Cancer Programm Associazione Italiana per le
Conflicts of interest: none.

1 Devesa SS, Fears T. Non-Hodgkin's lymphoma time trends: United States and international data. Cancer Res United States and

2 Levine PH, Hoover RN. The emerging epidemic of non-Hodgkin's lymphoma. Cancer Res. 1992;52:5425$574 \mathrm{~s}$

3 Coleman MP, Esteve J, Damiecki P, et al. Trends in cancer incidence and mortality. Sci Publ no 121. Lyon: International Agency for Resarch on Cancer, 1993

4 Parkin DM, Muir CS, Whelan SL, et al, eds. Cancer incidence in five continents. Volume VII. Sci Publ no 143. Lyon, International Agency for Research on Cancer, 1997.
5 Kersey JH, Shapiro RS, Filipovich AH. Relationship of immunodeficiency to lymphoid malignancy. Pediatr Infect Dis f 1988; 7:S10-12.

6 Johnson CC, Wilcosky T, Kvale T, et al. Cancer incidence among an HIV-infected cohort. Am f Epidemiol 1997;146: $470-5$.

7 Cartwright R, McNally R, Staines A. The increasing incidence of non-Hodgkin's lymphoma (NHL): the possible role of sunlight. Leuk Lymphoma 1994;14:387-94.

8 Vineis P, D'Amore F, and Working Group on the Epidemiology of Hematolymphopoietic Malignancies in Italy. The role of occupational exposure and immunodeficiency in B-cell malignancies. Epidemiology 1992;3:266-70.

9 Scherr PA, Mueller NE. Non-Hodgkin's disease. In: Schottenfeld D, Fraumeni JF, eds. Cancer epidemiology and prevention. 2nd ed. New York: Oxford University Press, 1996.

10 Banks PM. Changes in diagnosis of non-Hodgkin's lymphomas over time. Cancer Res 1992;52:5453-5s

11 Mueller NE. Hodgkin's disease. In: Schottenfeld D, Fraumeni JF, eds. Cancer epidemiology and prevention. 2nd ed. New York: Oxford University Press, 1996.

12 Cortes J, Kurzrock R. Interleukin-10 in non-Hodgkin's lymphoma. Leuk Lymphoma 1997;26:251-9.

13 D'Elios MM, Manghetti M, Almerigogna F, et al. Different cytokine profile and antigen-specificity repertoire in Helicobacter pylori-specific T cell clones from the antrum of chronic gastritis patients with or without peptic ulcer. Eur F Immunol 1997;27:1751-5.

14 Shirikawa T, Enomoto T, Shimazu S, et al. The inverse association between tuberculin responses and atopic disorder. Science 1997;275:77-9.

15 Holt PG, Yabuhara A, Prescott S, et al. Allergen recognition in the origin of asthma. Ciba Found Symp 1997;206:35-49.

16 Barrios C, Brawand P, Berney M, et al. Neonatal and early life responses to various forms of vaccine antigens qualitatively differ from adult responses: predominance of a Th2biased pattern which persists after adult boosting. Eur $\mathcal{F}$ Immunol 1996;26:1489-96.

17 Lucey DR, Clerici M, Shearer GM. Type 1 and Type 2 cytokine dysregulation in human infections, neoplastic and inlammatory diseases. Clin Microbiol Rev 1996;9:532-62.

18 Rook GAW, Stanford JL. Give us this day our daily germs. Immunol Today 1998;19:113-16.

19 Guthenson N, Shapiro D. Social class risk factors among children with Hodgkin's disease. Int f Cancer 1982;30:4335.

20 Cohen BM, Smetana HE, Miller RW. Hodgkin's disease. Long survival in a study of 388 World War II army cases. Cancer 1964;17:856-66.

21 Guthenson N, Cole P. Epidemiology of Hodgkin's disease in the young. Int $\mathcal{f}$ Cancer 1977;19:595-604.

22 Guthenson N. Social class and age at diagnosis of Hodgkin's diseases: new epidemiologic evidence on the "two-disease hypothesis". Cancer Treat Rep 1982;66: 689-95.

23 Paffenbarger RS, Wilson VO. Previous tonsillectomy and current pregnancy as they affect the risk of poliomyelitis attack. Ann NY Acad Sci 1955;61:856-68.

24 Poskanzer DC. Tonsillectomy and multiple sclerosis. Lancet 1965;ii: $1264-6$.

25 Cookson WOCM, Moffatt MF. Asthma: an epidemic in the absence of infection? Science 1997;265:41-2

26 Kramer U, Heinrich J, Wjst M, et al. Age at entry to day nursery and allergy in later childhood. Lancet 1999;353: $450-4$.

27 Wald ER, Dashefsy B, Byers C, et al. Frequency and severity of infections in day care. 7 Pediatr 1988;112:540-6.

28 Osterholm MT. Infectious diseases in child care: an overview. Pediatrics 1994;94:987-90.

29 The non-Hodgkin's lymphoma pathologic classification project: National Cancer Institute sponsored study of classifications of non-Hodgkin's lymphomas. Summary and description of a working formulation for clinical usage. Cancer 1982;49:2112-35.

30 Vineis P, Crosignani P, Sacerdote C, et al. Haematopoietic cancer and medical history: a multicentre case-control study. $\mathcal{F}$ Epidemiol Community Health 2000;54:431-6.

31 Breslow NE, Day NE, eds. Statistical methods in cancer research. Vol 1. The analysis of case-control studies. IARC Scientific Publication no 32. Lyon: International Agency for Reseach on Cancer, 1980.

32 The International Study of Asthma and Allergies in Childhood (ISAAC) Steering Committee: worldwide variations in the prevalence of asthma symptoms: the International Study of Asthma and Allergies in Childhood (ISAAC). Eur Respir f 1998;12:315-35.

33 Moyes CD, Waldon J, Dharmalingam R, et al. Respiratory symptoms and environmental factors in schoolchildren in the Bay of Plenty. NZ Med f 1995;108:358-61.

34 Lindberg C, Andersen O, Vahlne A, et al. Epidemiological investigation of the association between infectious mononucleosis and multiple sclerosis. Neuroepidemiology 1991; 10:62-5. 